\title{
Influence of Reckless Jaywalking on Urban Road Traffic Capacity
}

\author{
Xingli Li ${ }^{1, *}$, Fang Guo ${ }^{1}$ and Hua Kuang ${ }^{2}$ \\ ${ }^{I}$ School of Applied Science, Taiyuan University of Science and Technology, Taiyuan, 030024, China; ${ }^{2}$ College of Physi- \\ cal Science and Technology, Guangxi Normal University, Guilin, 541004, China
}

\begin{abstract}
A modified cellular automaton (CA) model based on the NaSch model is proposed to simulate typical reckless jaywalking which can cause serious traffic jam and even traffic accident. With a view to assess traffic psychology, walking characteristics such as customary activity, randomness, conformity and tendency are analyzed. Two parameters reflecting the influence degree of human factor are introduced with the global consideration of the above walking characteristics. The numerical simulations are carried out under periodic boundary conditions. Different cases including reckless jaywalking at a fixed position and other stochastic positions are taken into account. The relationship between the traffic states and the weight of human factor is investigated. The corresponding fundamental diagrams and the spatio-temporal patterns are given. Furthermore, the formation mechanism of traffic jam is explored and the measures for decreasing the negative influence of human factors are discussed.
\end{abstract}

Keywords: Cellular automaton model, Reckless jaywalking, Traffic flow, Traffic psychology.

\section{INTRODUCTION}

Recently, traffic has become a global exasperating problem, and the increasing traffic jams and traffic pollution are exerting great pressure on the society and are causing enormous economic losses. Many researchers from different disciplines have been trying to understand the fundamental principals governing the traffic based on three different approaches, namely: the macroscopic, mesoscopic and microscopic description [1-3]. Within the conceptual framework of the microscopic approach, the cellular automata (CA) has become popular for modeling traffic because it is conceptually simple with high computer efficiency. Much effort has been concentrated on stochastic CA models of traffic flow first proposed by Nagel and Schreckenberg [4] and subsequently studied by other authors using a variety of techniques. Now this model has been widely used in investigating different kinds of traffic situations such as blockages, multi-lanes traffic, mixed traffic, etc [5-9].

As we know, another significant reason contributing to jams is human influences such as reckless jaywalking, nonmotor vehicles moving on the motorway, motor vehicles changing lanes or making a U-turn randomly, taxies stopping casually, etc [10]. A recent survey has revealed that the influence of human factors on traffic has exceeded over $30 \%$ [11]. Moreover, the traffic nodes and blockage from human factors are becoming an increasingly key reason influencing the traffic. Especially in China, with large population and mixed traffic, pedestrians compete with vehicles to cross the road, and thus the influence of human factors on traffic is more serious.

*Address correspondence to this author at the School of Applied Science, Taiyuan University of Science and Technology, Taiyuan, 030024, China; Tel/Fax: 13466868371; E-mail: lixingli80@163.com
The answer to this question requires deep investigation. Unfortunately, most of the literature has been focused on the interaction between vehicles and other vehicles, or pedestrians and other pedestrians, while the effect of human factors on vehicles has rarely been discussed. In this paper, our primary aim was to propose a modified NaSch model by analyzing the characteristics of human factors from traffic psychology, which can contribute to a better understanding of the properties of traffic. The study focused on the reckless jaywalking in branch of main roads, where the walkers' behaviors are more casual. Once a traffic jam begins, it quickly expands to urban street networks.

This paper is organized as follows: Section 2 is devoted to explain the model. In Sections 3 and 4, we have presented the main results obtained by simulation with a detailed discussion. Finally, a general conclusion is drawn.

\section{MODEL}

\subsection{Behavior Analysis of Reckless Jaywalking}

The citizens' consciousness degree of obeying traffic regulations has a direct effect on the vehicles' speed and road capacity. Investigations show that nearly all traffic contributors including drivers, cyclists and walkers understand traffic from the viewpoint of their self-convenience. The main aim is to save time and strength, thus decreasing time in transit and physical exertion. Traffic psychology, as a branch of psychology, is primarily concerned with the study of behavior of people driving on the roads and how that behavior influences their actions on the road. It aims to shed some light on some of our more interesting behavior tendencies and to improve safety and comfort and increase efficiency of the transport system. It tells us that though people's behavior is complicated, there are some common characteristics as follows: 
First, the behavior of reckless jaywalking is a customary activity, often characterized by crossing the road from not the sidewalk but other positions for his/her convenience.

Second, the behavior is random, i.e., irregular crossing position, crossing time and the time interval for next crossing. Compared with traffic lights, the crossing position is unfixed and the time including the crossing time and the time interval for the next crossing are more stochastic.

Besides, another important characteristic is conformity or the following effect. In many cases, one walker's jaywalking can lead to blind following. Consequently, the crossing time increases inevitably.

\subsection{The CA Model Considering Jaywalking}

Generally, the pedestrian's crossing time is one of the most important factors affecting traffic capacity. Stochastic variables $t$ and $t 1$ are introduced to describe the crossing time and the time interval for the next crossing, respectively. They reflect the influence degree of the human factors. Some modifications based on $\mathrm{NaSch}$ model are performed as follows:

1) The jaywalking behavior is added to the NaSch model. It is set to one and some lattices randomly.

2) The moving rules for normal vehicles can change when confronted with jaywalking. Let dstop denote the empty sites in front of the nth vehicle with the crossing position. When $d_{\text {stop }} \in[0,5], v_{n}(t+1) \rightarrow 0$. In other cases, vehicles move as updating rules of the NaSch model. Generally, in urban areas, pedestrian behavior is always more "aggressive" than vehicles, which means that the vehicles always decelerate voluntarily while pedestrians always cross the street as soon as possible when they feel the situation is safe. Considering safety, theoretically, we assume that the vehicle's velocity decelerates to zero once the distance between the vehicle and pedestrian is not more than $5(\mathrm{~m} / \mathrm{cm}$ ?). (AUTHOR: The unit is missing. Please provide that)

\section{SIMULATION AND RESULTS}

Numerical simulations are performed according to the above updating rules under the periodic boundary condition. A one-dimensional lattice of $L$ sites and vehicles moving uni-directionally is considered. Each site is set to be $7.5 \mathrm{~m}$ long, $L$ to be 200 , one time step to be $1 \mathrm{~s}$, being the order of the reaction time for humans, and the maximum velocity to be $v_{\max }=3$, corresponding to $81 \mathrm{~km} / \mathrm{h}$. In order to characterize the behavior of the model, globally the macroscopic quantities are determined, including the global density $\rho$, the mean speed $V$ and the mean flow $J$ defined as:

$\rho=N / L$

$V=\frac{1}{T} \sum_{t=t_{0}}^{t=T+t_{0}-1} \frac{1}{N} \sum_{n=1}^{N} v_{n}(t)$

$J=\rho V$

In numerical simulation, the first $5^{\prime} 10^{4}$ time-steps of each run are put away in order to remove the transient ef- fects, and then the data are recorded in successive $1^{\prime} 10^{4}$ time-steps. The mean velocity is obtained by averaging over 30 runs of simulations. Two typical situations are considered.

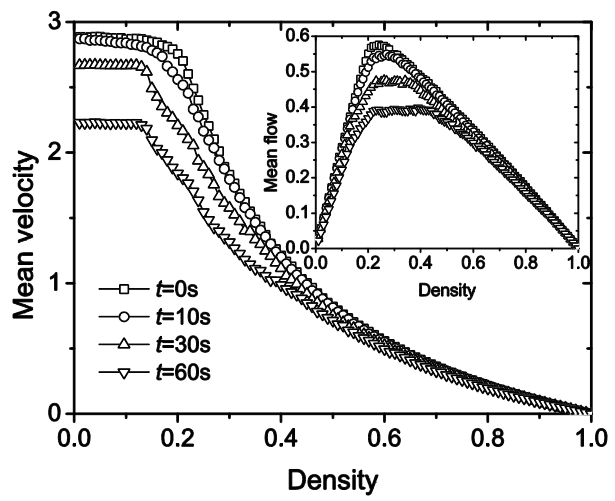

(a)

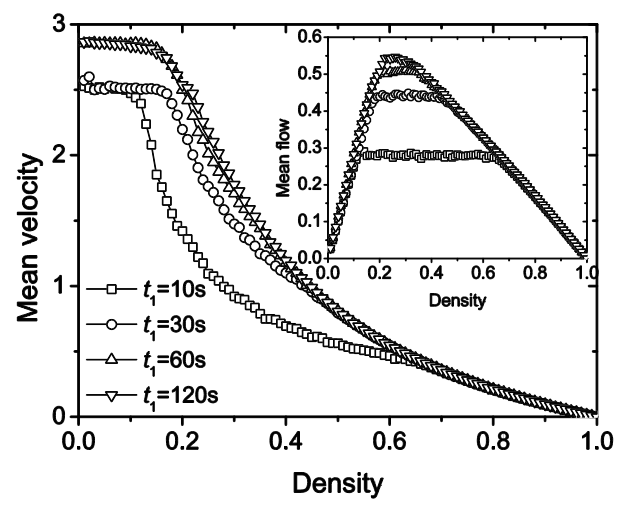

(b)

Fig. (1). The fundamental diagrams with different crossing time (a) and with different time interval for the next crossing (b)

Case 1: Jaywalking at a fixed crossing position

The fundamental diagram for different crossing times $t$ with the fixed time interval for the next crossing $t_{1}=120 \mathrm{~s}$ is shown in Fig. (1a). As $t>0$, the flow increases linearly at low densities, but it is lower than that of $t=0$. Then the flux saturates at the first critical density and keeps a constant value till the occurrence of the second critical density. Moreover, as $t$ increases, the saturated flow plateau decreases. As $\rho$ is larger than the second critical density, the flow decreases with the increase in density. Especially, when $t=60 \mathrm{~s}$, the saturated flow declines to $70 \%$. Considering the effect of crossing time, with the increase in $t$, the flow decreases except for the consistence in a higher density $(\rho>0.6)$. The first critical density decreases but the second critical density increases with the increase in $t$. This means for a larger crossing time $t$, the density range corresponding to the saturated flow also increases. A possible explanation is that with the increase in the crossing time, more and more vehicles cannot move at an expected speed, which leads the critical density from free flow to saturated flow to a decrease. At the same time, the decreasing flux requires more vehicles for the occurrence of congested phase, so the second critical density increases. The results are in agreement with real traffic. 


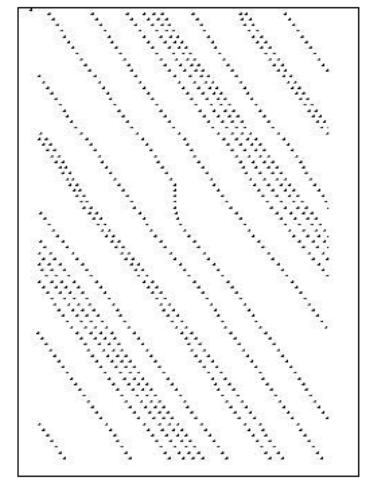

(a) $\rho=0.05, t=10 \mathrm{~s}$

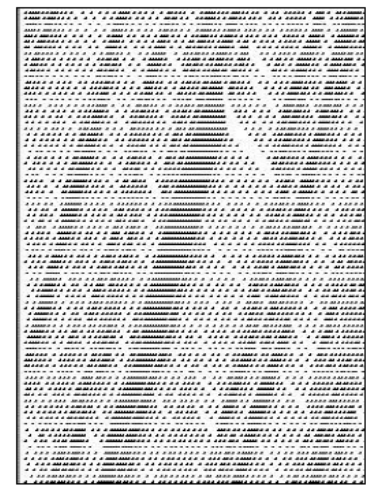

(c) $\rho=0.4, t=10 \mathrm{~s}$

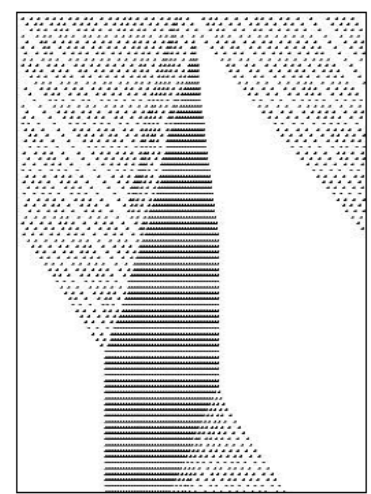

(e) $\rho=0.2, t=60 \mathrm{~s}$

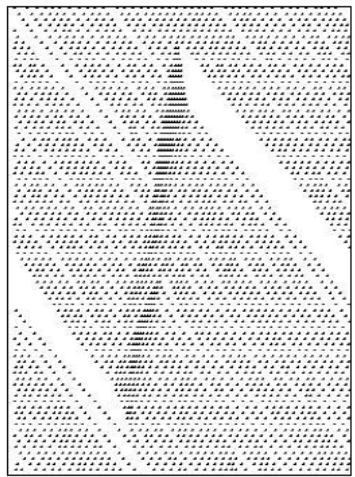

(b) $\rho=0.2, t=10 \mathrm{~s}$

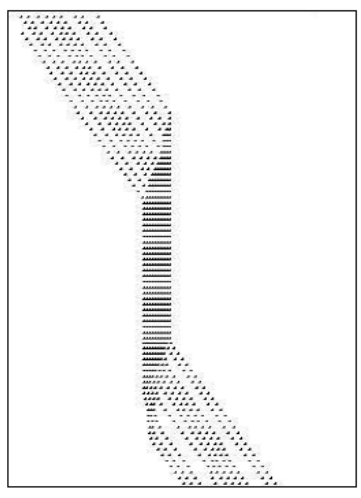

(d) $\rho=0.05, t=60 \mathrm{~s}$

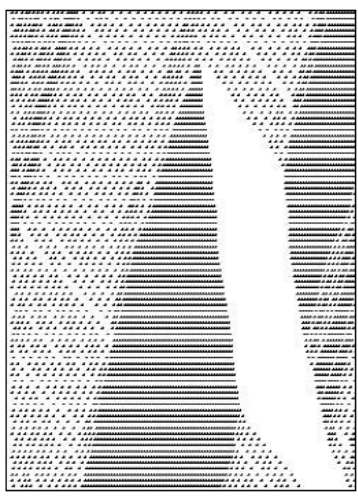

(f) $\rho=0.4, t=60 \mathrm{~s}$

Fig. (2). The spatiotemporal patterns obtained at $t_{1}=120 \mathrm{~s}$.

The spatiotemporal patterns, with different densities $\rho=0.05,0.2,0.4$ and crossing times $t=10 \mathrm{~s}, 60 \mathrm{~s}$, are shown in Fig. (2), where $t_{1}=120 \mathrm{~s}$ is constant. The abscissa denotes the spatial position, i.e. the position of the cells in the lane, while the ordinate denotes the simulation time steps. Black points denote vehicles while white points represent blank spaces. When the crossing position is at location 100 on the abscissa, the vehicle moves left to right. In the spatiotemporal map, jaywalking leads to a line of waiting vehicles in front of the pedestrians, which evolves with time as the black area in the map. The blank area behind the crossing position is the vacant area when some walkers begin to cross the road. Far from the crosswalk, the vehicle density tends to be sparse and stable. Comparison of Fig. (2) under different densities and crossing times shows how the map characteristics change with $\rho$ and $t$. Even in low density, the increasing crossing time leads to a big jam.

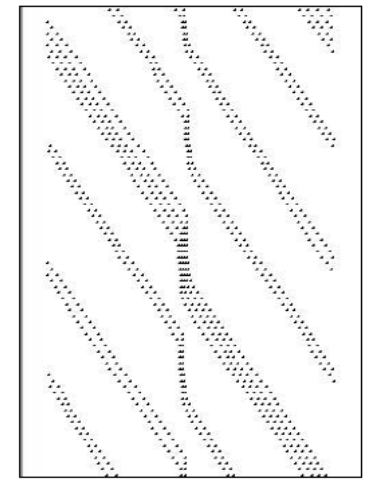

(a) $\rho=0.05, t_{1}=10 \mathrm{~s}$

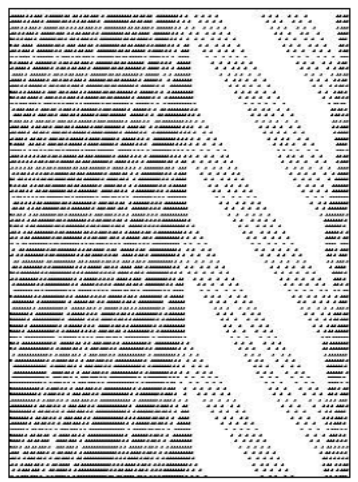

(c) $\rho=0.4, t_{1}=10 \mathrm{~s}$

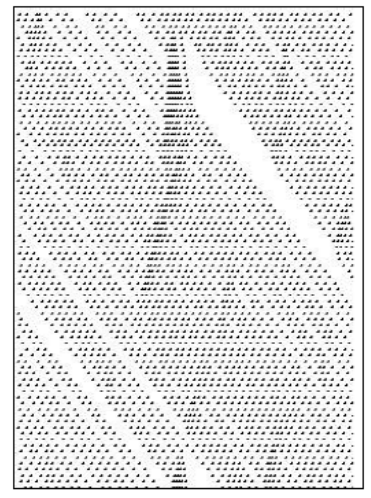

(e) $\rho=0.2, t_{1}=60 \mathrm{~s}$

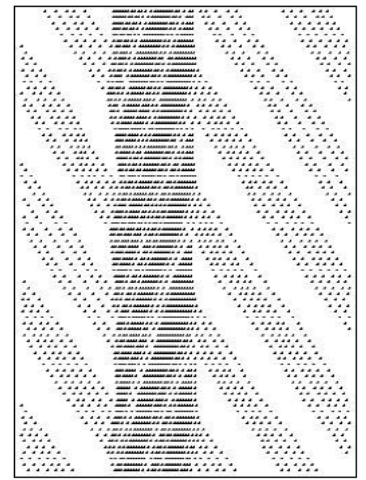

(b) $\rho=0.2, t_{1}=10 \mathrm{~s}$

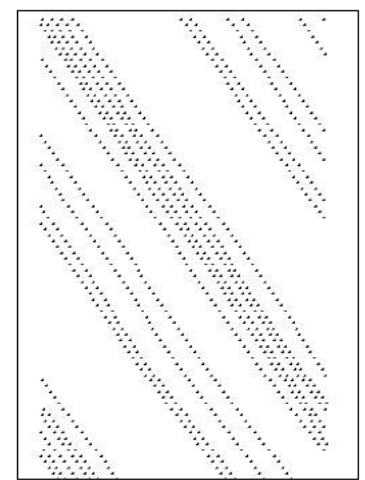

(d) $\rho=0.05, t_{1}=60 \mathrm{~s}$

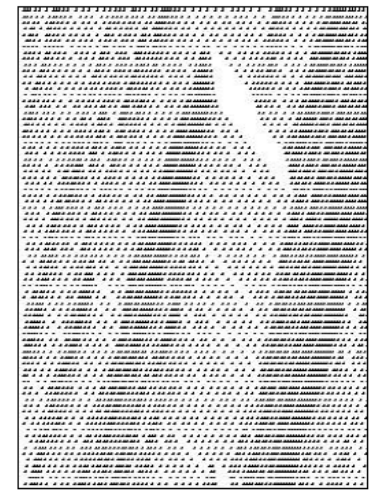

(f) $\rho=0.4, t_{1}=60 \mathrm{~s}$
Fig. (3). The spatiotemporal patterns obtained at $t=10 \mathrm{~s}$.

The fundamental diagram with fixed $t(t=10 \mathrm{~s})$ and variable $t_{1}$ is given in Fig. (1b). With the increasing time interval of crossing, the saturated value of flow and the mean velocity both increase obviously. When the crossing frequency is high, e.g. $t_{1}=10 \mathrm{~s}$, the saturated flow is only 0.28 , declining by $50 \%$. A conclusion can be drawn that frequent crossing has a more negative influence on the traffic flow compared with the increasing crossing time in the low density. The spatiotemporal patterns under different $\rho$ and $t_{1}$ are shown in Fig. (3). It is clear that the increase in crossing frequency makes the vehicle accelerate and decelerate more frequently. All these further validate the former fundamental diagram.

\section{Case 2: Reckless jaywalking at some stochastic positions}

In real traffic, the randomness of pedestrian's behavior is universal. This randomness mainly includes stochastic crossing position, different crossing times under different positions and different time intervals for the next crossing, 


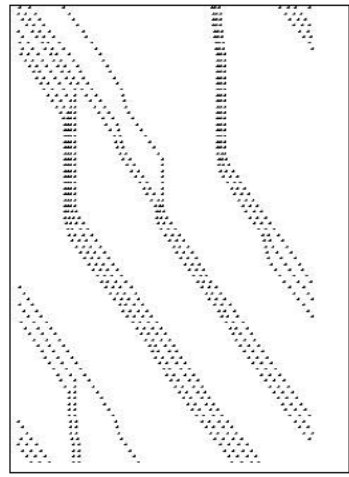

(a) $\rho=0.05$

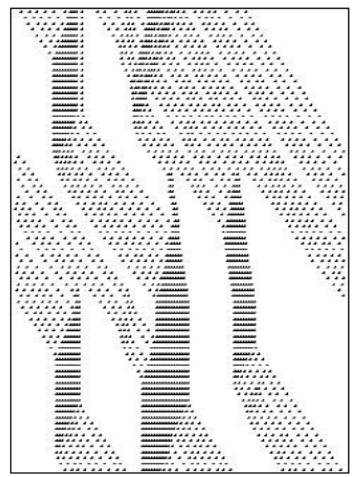

(b) $\rho=0.2$

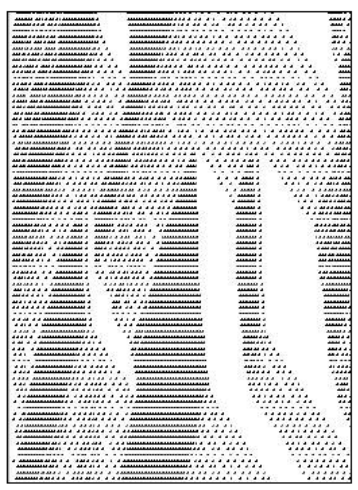

(c) $\rho=0.4$

Fig. (4). The spatiotemporal patterns with five random crossing positions. etc. Here, $t$ is selected randomly in a range $[10,30]$ and $t_{1}$ as given $[10,60]$. Fig. (4) shows the space time patterns with low, middle and high densities $\rho=0.05,0.2,0.4$. Due to various indefinite factors, the movement of vehicle is out of order. Compared with those in a fixed position, even in low density, the vehicle decelerates more often.

\section{OTHER ANALYSIS AND DISCUSSION}

In real traffic, how to reduce the influence of human factors is still tough work. To some extent, proper management strategies and road design would be helpful to ease traffic congestion. Three typical questions are worth further discussion.

1) Influence of crossing frequency. Under the same conditions, let the pedestrian cross the road in a long time or a short time but with high frequency, and then evaluate which one is better. Fig. (5a) shows the fundamental diagram under different crossing frequencies with constant total crossing time $t_{\text {total }}=120 \mathrm{~s}$ and time interval for the next crossing $t_{1 \text { total }}=240 \mathrm{~s}$. During the total time, the pedestrian would cross the road with different $t$ and $t_{1}$. The crossing time length directly determines the crossing frequency. At lower density $(\rho<0.05)$, both the vehicle flow and velocity with $t=10 \mathrm{~s}, 20 \mathrm{~s}$ are larger than that in other cases, which implies that the short crossing time has little influence on vehicle flow since the probability for the vehicle to decelerate or stop is reduced obviously. An interesting phenomenon is found that the velocity of $t=40 \mathrm{~s}$ is larger than that of $t=30 \mathrm{~s}$ although being still lower than that of $t=60 \mathrm{~s}, 120 \mathrm{~s}$; while $0.05<\rho<0.18$, its velocity drops distinctly, less than that of $t=30 \mathrm{~s}, 60 \mathrm{~s}, 120 \mathrm{~s}$.
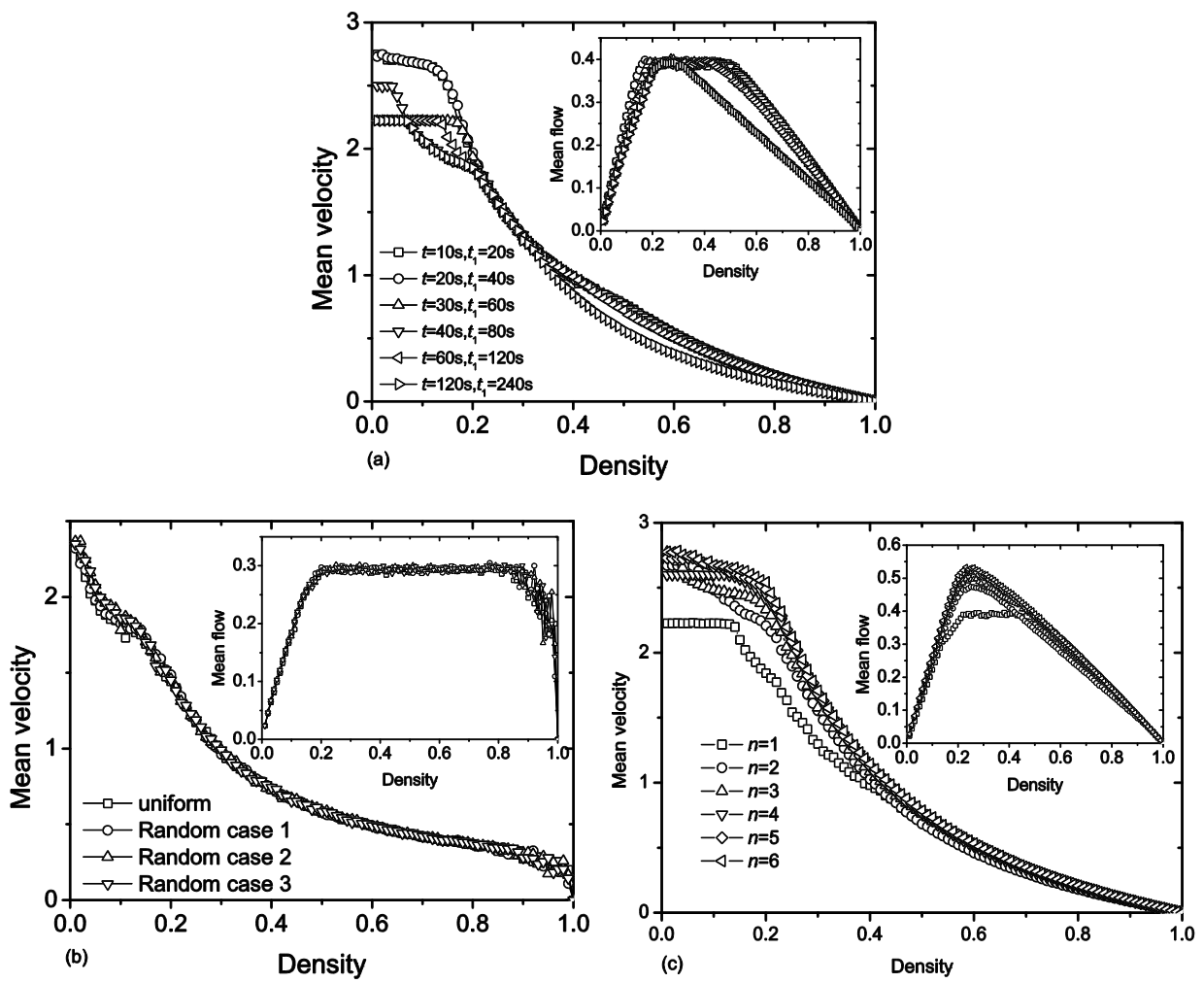

Fig. (5). The fundamental diagrams with different crossing frequencies (a), Uniform and random crossing positions (b) and different numbers of crossing positions (c) 
All these reflect low density region; traffic is under double influence of crossing time and frequency. In the middle and high density, mean flow and velocity of $t=120 \mathrm{~s}$ is much lower than that of others. Broadly, compared with the crossing frequency, the influence of crossing time of each cross on traffic capacity and efficiency is much greater, especially in crowded areas, so policies are needed to restrict the behaviors of jaywalking. For example, crossing the street partially under the guidance of traffic light or police may be required.

2) Influence of distribution of crossing position. Crossing positions can be random or uniform but the question arises as to which is more proper. The corresponding fundamental diagram is shown in Fig. (5b), where five crossing positions and three random cases are selected. It can be seen that the distribution of crossing position has little influence on the vehicle flow under fixed $n$. An important enlightenment to the road construction can be taken into consideration that at constant road length, the setting of the traffic light mainly depends on real circumstances instead of doing that apart same.

3) Influence of the number of crossing positions. Under the same conditions, let the pedestrian cross the road through a position or several positions within a short time but then which is better. Fig. (5c) presents the fundamental diagram under different crossing position numbers $n=1, \ldots, 6$ with fixed road length. Here, $t_{\text {total }}=60 \mathrm{~s}$ and $t_{1}=120 \mathrm{~s}$ remain constant. It can easily be seen that larger $n$ could lead to relatively larger flow and velocity, especially in low and middle density region, and the saturated flow plateau gradually disappears. In comparison with the case of $n=6$, the maximum flow of $n=1$ drops nearly by $30 \%$. On one hand, to some extent, the decreasing crossing time in every position reduces the possibility of brake or deceleration; on the other hand, vehicle starting time delay in a long queue is much more than that in a short queue. Whereas, it should be noted that in high density $(\rho>0.4)$, the mean velocity and flow of $n=2$ are a litter lower than in other cases, which means that in such case, it is quite necessary to consider the influence of both the crossing time and the number of positions.

\section{CONCLUSION}

Based on numerical investigation and discussion presented in this paper, some important conclusions can be drawn as follows: (1) The saturated flow appears in the fundamental diagram. Its value depends obviously on the increasing crossing time and crossing frequency, which lead to the sudden drop in mean velocity and flow; (2) In different densities, the influence of $t$ and $t 1$ on traffic is also different; (3) For the constant total crossing time and time interval for the next crossing, the increase in crossing frequency expands the density range corresponding to the saturated flow; especially in crowded areas, the increase in single crossing time can decrease the actual capacity of road obviously; (4) The distribution of crossing position, random or uniform, shows little influence on the traffic; (5) Generally, under the same conditions, a decentralized crossing at several positions is superior to a centralized crossing at a single position in a long time.

Finally, the method and some results can be extended to other human factors. We hope that our results here can be useful to the signal timing dial and road construction.

\section{CONFLICT OF INTEREST}

The authors confirm that this article content has no conflict of interest.

\section{ACKNOWLEDGEMENTS}

This work is supported by the National Natural Science Foundation of China (Nos. 10902076, 11262005 and 11162019), the Natural Science Foundation of Shanxi Province (No. 2010011004), the Top Young Academic Leaders of Higher Learning Institutions of Shanxi, Guangxi Natural Science Foundation (No. 2012GXNSFBA053002) and the Key Project of Education Department of Guangxi (No. 201202ZD015).

\section{REFERENCES}

[1] D. Chowdhury, L. Santen, and A. Schadschneider, "Statistical physics of vehicular traffic and some related systems," 2000 Phys. Rept., vol. 329, pp. 199-329, 2000.

[2] D. Helbing, "Traffic and related self-driven many-particle systems," Rev. Mod. Phys., vol. 73, pp. 1607-1141, 2001.

[3] S. L. Paveri-Fontana, "On Boltzmann-like treatments for traffic flow: a critical review of the basic model and an alternative proposal for dilute traffic analysis," Trans. Res., vol. 9, pp. 225-235, 1975.

[4] K. Nagel, and M. Schreckenberg, "A cellular automaton model for freeway traffic," J. Phys. I (France), vol. 2, pp. 2221-2229, 1992.

[5] S. Maerivoe, and B. D. Moor, "Cellular automata models of road traffic," Phys. Rep., vol. 419, pp. 1-64, 2005.

[6] M. M. Pedersen, P. T. Ruhoff, "Entry and an alternative proposal for dilute traffic analysis," Phys. Rev. E, vol. 65, pp. 056705, 2002.

[7] B. Jia, R. Jiang, and Q. S. Wu, "Traffic behavior near an off ramp in the cellular automaton traffic model," Phys. Rev. E, vol. 69, pp. 056105, 2004.

[8] H. Ez-Zahraouya, K. Jetto, and A. Benyoussef A, "The effect of mixture lengths of vehicles on the traffic flow behaviour in onedimensional cellular automaton," Eur. Phys. J. B, vol. 40, pp. 111117, 2004.

[9] J. P. Meng, S. Q. Dai, L. Y. Dong, and J. F. Zhang, "Cellular automaton model for mixed traffic flow with motorcycles," Physica $A$, vol. 380, pp. 470-480, 2007.

[10] J. Shao, and B. He, "The reasons and countermeasures' analysis of road traffic jams in Beijing," Road Traffic \& Safety, vol. 8, pp. 3944, 2001. (in Chinese)

[11] X. K. Fan, "Study on traffic jams in cities," China Public Security, vol. 1, pp. 48-51, 2007. (in Chinese)

[12] S. R. Fan. Course in Traffic Psychology. Chinese People‘s Public Security University Press, 2005. (in Chinese)

\footnotetext{
Received: September 16, 2014

Revised: December 23, 2014

Accepted: December 31, 2014

(C) Li and et al.; Licensee Bentham Open.

This is an open access article licensed under the terms of the Creative Commons Attribution Non-Commercial License (http://creativecommons.org/licenses/by-nc/4.0/) which permits unrestricted, non-commercial use, distribution and reproduction in any medium, provided the work is properly cited.
} 